\title{
Crossing length scales
}

\author{
The Nobel Prize in Chemistry 2013 celebrates the use of computer simulations to model complex \\ chemical systems using multiscale approaches. Taken in a broad sense, these ideas and techniques \\ extend well beyond chemistry.
}

Even by its own standards, the announcement by the Royal Swedish Academy of Sciences that the 2013 Nobel Prize in Chemistry would be awarded to Martin Karplus, Michael Levitt and Arieh Warshel "for the development of multiscale models for complex chemical systems" was remarkably vague ${ }^{1}$. Its significance, however, was immediately recognized by a community of chemists that has long become accustomed to and benefited enormously from the numerical models and software that in effect allow for virtual experiments to be carried out on computers.

The key to this year's announcement is the word 'multiscale'. Computational chemistry has, of course, already been recognized by the Nobel committee, who awarded the 1998 chemistry prize to Walter Kohn and John Pople. Yet this year's award touches on a different complex problem: the interface between quantum and classical mechanics. On the length scale of atoms and small molecules, the behaviour of the electrons in their orbitals is dictated by the laws of quantum mechanics. Ideally, one would like to simulate larger biomolecules in this way as well, but this remains out of reach even for the most powerful of modern computers. Therefore, a pragmatic approach is to model larger systems, such as proteins, as spheres connected to each other by classical springs. The difficulty lies in knowing how and when to coarse-grain the quantum mechanical effects without compromising predictive accuracy.

In the early 1970s, at the time when Warshel and Karplus were working to calculate the electronic spectra of conjugated organic molecules ${ }^{2}$, this choice was imposed by severe limitations in computational power - a typical computer would have been six orders of magnitude less powerful than an average laptop today. By 1975, Levitt and Warshel had reported computer simulations of proteins folding ${ }^{3}$, and a year later they had constructed a general scheme for modelling enzymatic reactions ${ }^{4}$. The key insight was to simulate the molecular dynamics of the majority of the biomolecule using Newton's classical equations of motion, but to resort to quantum chemical methods for the active sites in which the chemical reactions actually occur. Coupling these two parts of the calculation, both to each other and to the environment

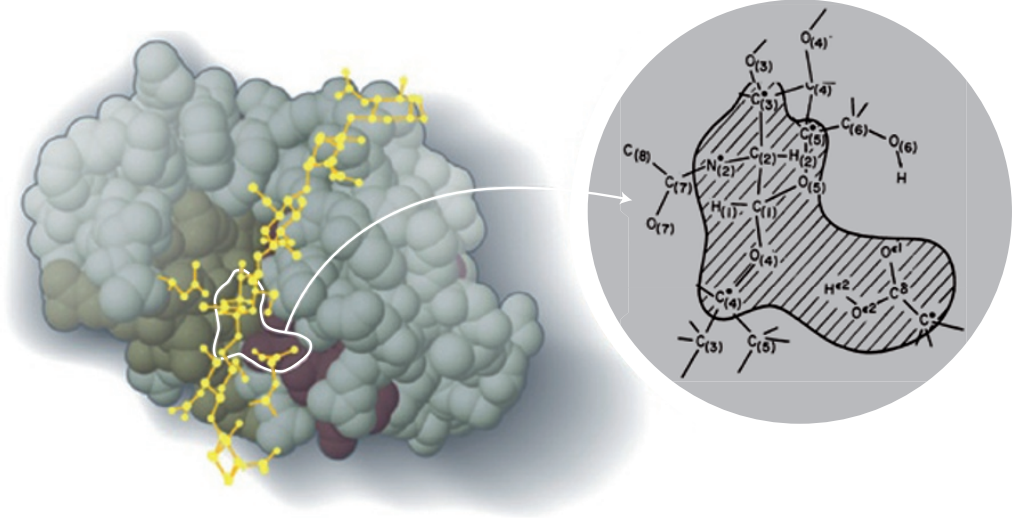

The protein lysozyme (left) cleaves glycosidic bonds (yellow). Multiscale models bring together detailed quantum-mechanical calculations of the reaction sites (right) with classical dynamics to model the rest of the protein. Figures reproduced with permission from: left, (c) Johan Jarnestad/The Royal Swedish Academy of Sciences; right, ref. 4, (c) 1976 Elsevier.

surrounding the biomolecule, remained a tricky but ultimately tractable problem.

One profound implication of these early calculations was to replace the idea that proteins were rigid structures; it is now well established that fluctuations are actually crucial for understanding their function. And with the spectacular growth in computer power witnessed since the 1970s, researchers have been able to model increasingly complex systems with ever increasing accuracy. Moreover, the importance of reliable and efficient computer software to run the simulations was recognized from the earliest days. Indeed, Karplus was instrumental in developing CHARMM (Chemistry at Harvard Macromolecular Mechanics), a computer program that is still used by a wide community of researchers.

It is striking how widespread and successful this approach has become in most scientific disciplines. In materials science, computational modelling has become a central pillar of the discipline, equal in standing to experiment and theory. Codes are developed by communities of researchers across different institutions and countries, and they are used to address some of the most challenging problems in fields, ranging from study of mechanical properties ${ }^{5}$ to device physics ${ }^{6}$. Increasingly, the huge data sets generated both by experiments and simulations give rise to new challenges that are best met through collaboration and sharing ${ }^{7}$.
But there is also a broader, more fundamental theme that is implicit in this year's Nobel prize, namely, the value of cross-disciplinary research. The early studies examining the boundary between quantum chemistry and molecular dynamics had their roots in chemistry, but their influence is most keenly felt in biochemistry and biology. One can only admire the ingenuity of these early pioneers, who remained unfazed by the sheer complexity of the problems they chose to address. This mentality is now pervasive in materials science, and computational models are the tool with which basic understanding on a microscopic length scale is translated into predictive power on a macroscopic one.

Just like the pioneering multiscale calculations bridged the gap between quantum and classical mechanics, the general concept of multiscale modelling has crossed over into various disciplines. With increasingly sophisticated algorithms and ever increasing computer power, the template put forward by Karplus, Warshel and Levitt will doubtless continue to be productive for years to come. $\square$

\footnotetext{
References

1. The Nobel Prize in Chemistry 2013; http://www.nobelprize.org/ nobel_prizes/chemistry/laureates/2013 (30 October 2013).

2. Warshel, A. \& Karplus, M. J. Am. Chem. Soc. 94, 5612-5625 (1972).

3. Levitt, M. \& Warshel, A. Nature 253, 694-698 (1975).

4. Warshel, A. \& Levitt, M. J. Mol. Biol. 103, 227-249 (1976).

5. Yip, S. \& Short, M. P. Nature Mater. 12, 774-777 (2013).

6. Mesta, M. et al. Nature Mater. 12, 652-658 (2013).

7. Nature Mater. 12, 173 (2013).
} 